Acta Crystallographica Section E

Structure Reports

Online

ISSN 1600-5368

\section{T. Seethalakshmi, ${ }^{\mathrm{a}}$ A.}

Puratchikody, ${ }^{\text {b }}$ Daniel E. Lynch, ${ }^{c}$

P. Kaliannan ${ }^{\mathrm{a}}$ and $\mathrm{S}$.

Thamotharan $^{\mathrm{d}}$ *

${ }^{a}$ School of Physics, Bharathidasan University, Tiruchirappalli 620 024, India, bepartment of Pharmacy, Bharathidasan University of

Technology, Bharathidasan University, Tiruchirappalli 620 024, India, 'Faculty of Health and Life Sciences, Coventry University, Coventry CV1 5FB, England, and Molecular Biophysics Unit, Indian Institute of Science,

Bangalore 560 012, India

Correspondence e-mail: thamu_as@yahoo.com

\section{Key indicators}

Single-crystal X-ray study

$T=120 \mathrm{~K}$

Mean $\sigma(\mathrm{C}-\mathrm{C})=0.004 \AA$

$R$ factor $=0.041$

$w R$ factor $=0.103$

Data-to-parameter ratio $=8.7$

For details of how these key indicators were automatically derived from the article, see http://journals.iucr.org/e.

\title{
2-(2-Methylphenyl)-4,5-diphenyl-1 H-imidazole
}

In the title molecule, $\mathrm{C}_{22} \mathrm{H}_{18} \mathrm{~N}_{2}$, all bond lengths and angles are normal. Intermolecular $\mathrm{N}-\mathrm{H} \cdots \mathrm{N}$ hydrogen bonds with an $\mathrm{N}$...N distance of 2.933 (2) $\AA$, link the molecules into chains running along the $c$ axis. The crystal packing is further stabilized by van der Waals forces.

\section{Comment}

Several heterocyclic compounds with aryl substituents have previously been reported to exhibit anti-inflammatory activity in animals (Almirante et al., 1965; Marchetti et al., 1968). Of the various polyaryl heterocycles, certain 4,5-diphenyl-2substituted imidazoles exhibited anti-inflamatory activity comparable to phenylbutazone in the carrageenan rat paw edema test (Lombardino \& Wiseman, 1974). In view of this importance, we report here the crystal structure of the title compound, (I), which is a 4,5-diphenyl-2-substituted imidazole derivative.

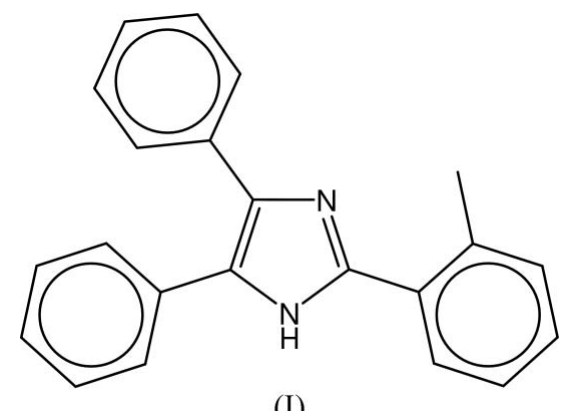

(I)

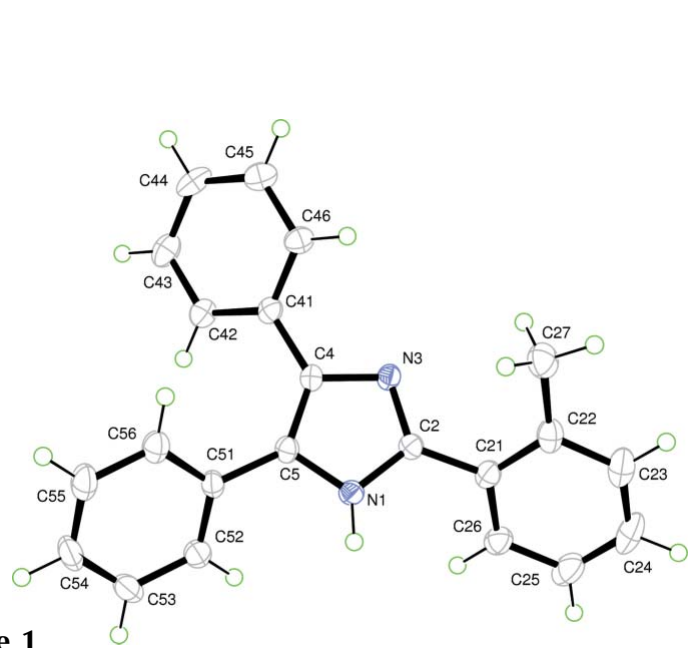

Figure 1

View of (I), showing the atom-labelling scheme and displacement ellipsoids at the $50 \%$ probability level. $\mathrm{H}$ atoms are represented by circles of arbitrary radii.
Received 26 May 2006 Accepted 2 June 2006 
The bond lengths and angles in (I) (Fig. 1) are in good agreement with the literature values (Allen et al., 1987). The imidazole ring makes dihedral angles of 41.2 (1), 31.5 (1) and 41.7 (1) ${ }^{\circ}$ with the C21-C26, C41-C46 and C51-C56 aromatic rings, respectively. In the solid state, intermolecular $\mathrm{N}-\mathrm{H} \cdots \mathrm{N}$ hydrogen bonds (Table 1) link the molecules into $C(4)$ chains (Bernstein et al., 1995) running along the $c$ axis. The crystal packing (Fig. 2) is further stabilized by van der Waals forces.

\section{Experimental}

A mixture of benzil $(5.25 \mathrm{~g}, 0.025 \mathrm{~mol})$, ammonium acetate $(10 \mathrm{~g}$, $0.129 \mathrm{~mol})$ and 2-methylbenzaldehyde $(0.018 \mathrm{~mol})$ in glacial acetic acid $(50 \mathrm{ml})$ was heated under reflux for $1-2 \mathrm{~h}$. The product was recrystallized from aqueous ethanol (yield 80\%, m.p. 484-486 K).

\section{Crystal data \\ $\mathrm{C}_{22} \mathrm{H}_{18} \mathrm{~N}_{2}$ \\ $M_{r}=310.38$ \\ Monoclinic, $C c$ \\ $a=10.7538(5) \AA$ \\ $b=19.3999$ (9) $\AA$ \\ $c=8.7900(3) \AA$ \\ $\beta=112.886(2)^{\circ}$ \\ $V=1689.44(13) \AA^{3}$}

$Z=4$

$D_{x}=1.220 \mathrm{Mg} \mathrm{m}^{-3}$

Mo $K \alpha$ radiation

$\mu=0.07 \mathrm{~mm}^{-1}$

$T=120$ (2) K

Rod, colourless

$0.36 \times 0.06 \times 0.04 \mathrm{~mm}$

\section{Data collection}

Nonius KappaCCD area-detector diffractometer

$\varphi$ and $\omega$ scans

Absorption correction: multi-scan (SADABS; Sheldrick, 2003)

$T_{\min }=0.745, T_{\max }=0.927$

(expected range $=0.801-0.997)$

\section{Refinement}

Refinement on $F^{2}$

$R\left[F^{2}>2 \sigma\left(F^{2}\right)\right]=0.041$

$w R\left(F^{2}\right)=0.103$

$S=1.06$

1945 reflections

223 parameters

$\mathrm{H}$ atoms treated by a mixture of independent and constrained refinement
10671 measured reflections 1945 independent reflections 1744 reflections with $I>2 \sigma(I)$ $R_{\text {int }}=0.059$

$\theta_{\max }=27.5^{\circ}$

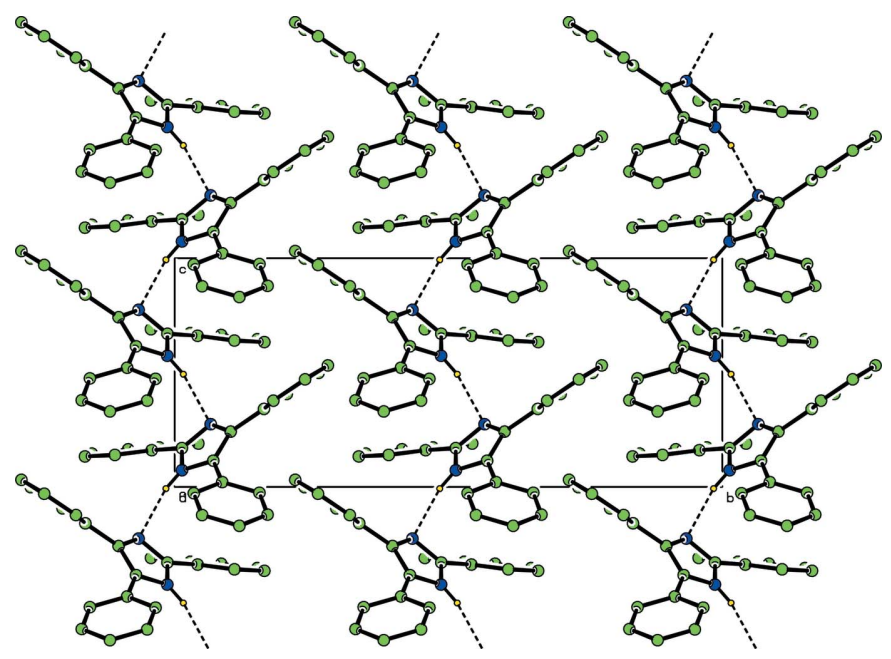

Figure 2

The crystal packing of (I), viewed along the $a$ axis. The intermolecular $\mathrm{N}-\mathrm{H} \cdots \mathrm{N}$ hydrogen bonds are shown as dashed lines. All the $\mathrm{H}$ atoms, except those involved in hydrogen bonding, have been omitted for clarity.

$1.2 U_{\text {eq }}(\mathrm{C})$. Owing to the absence of any significant anomalous scatterers in the molecule, the 1433 Friedel pairs were merged before the final refinement.

Data collection: COLLECT (Hooft, 1998); cell refinement: DENZO (Otwinowski \& Minor, 1997) and COLLECT; data reduction: DENZO and COLLECT; program(s) used to solve structure: SIR92 (Altomare et al., 1994); program(s) used to refine structure: SHELXL97 (Sheldrick, 1997); molecular graphics: ORTEP-3 for Windows (Farrugia, 1997) and PLATON (Spek, 2003); software used to prepare material for publication: SHELXL97.

The authors thank the EPSRC National Crystallography Service (Southampton, England). TS thanks Professors V. Parthasarathi, School of Physics and M. Nallu, School of Chemistry, Bharathidasan University, Tiruchirappalli, for their generous help.

\section{References}

Allen, F. H., Kennard, O., Watson, D. G., Brammer, L., Orpen, A. G. \& Taylor, R. (1987). J. Chem. Soc. Perkin Trans. 2, pp. S1-19.

Almirante, L., Polo, L., Mugnaini, A., Provinciali, E., Rugarli, P., Biancotti, A., Gamba, A. \& Murmann, W. (1965). J. Med. Chem. 8, 305-312.

Hydrogen-bond geometry $\left(\AA,^{\circ}\right)$.

\begin{tabular}{lllll}
\hline$D-\mathrm{H} \cdots A$ & $D-\mathrm{H}$ & $\mathrm{H} \cdots A$ & $D \cdots A$ & $D-\mathrm{H} \cdots A$ \\
\hline $\mathrm{N} 1-\mathrm{H} 1 \cdots \mathrm{N} 3^{\mathrm{i}}$ & $0.88(3)$ & $2.07(3)$ & $2.933(3)$ & $168(3)$ \\
\hline
\end{tabular}

Symmetry code: (i) $x,-y, z-\frac{1}{2}$.

The position of the amine $\mathrm{H}$ atom was determined from a difference Fourier map and refined freely along with its isotropic displacement parameter. The methyl $\mathrm{H}$ atoms were constrained to an ideal geometry $(\mathrm{C}-\mathrm{H}=0.98 \AA)$, with $U_{\text {iso }}(\mathrm{H})=1.5 U_{\text {eq }}(\mathrm{C})$, but were allowed to rotate freely about the $\mathrm{C}-\mathrm{C}$ bond. The remaining $\mathrm{H}$ atoms were placed in geometrically idealized positions $(\mathrm{C}-\mathrm{H}=$ $0.95 \AA$ ) and constrained to ride on their parent atoms with $U_{\text {iso }}(\mathrm{H})=$
Altomare, A., Cascarano, G., Giacovazzo, C., Guagliardi, A., Burla, M. C., Polidori, G. \& Camalli, M. (1994). J. Appl. Cryst. 27, 435.

Bernstein, J., Davis, R. E., Shimoni, L. \& Chang, N.-L. (1995). Angew. Chem. Int. Ed. Engl. 34, 1555-1573.

Farrugia, L. J. (1997). J. Appl. Cryst. 30, 565.

Hooft, R. W. W. (1998). COLLECT. Nonius BV, Delft, The Netherlands.

Lombardino, J. G. \& Wiseman, E. H. (1974). J. Med. Chem. 17, 1182-1188.

Marchetti, E., Mattalia, G. \& Rosnati, V. (1968). J. Med. Chem. 11, 1092-1093.

Otwinowski, Z. \& Minor, W. (1997). Methods in Enzymology, Vol. 276, Macromolecular Crystallography, Part A, edited by C. W. Carter Jr \& R. M. Sweet, pp. 307-326. New York: Academic Press.

Sheldrick, G. M. (1997). SHELXL97. University of Göttingen, Germany.

Sheldrick, G. M. (2003). SADABS. Version 2.10. Bruker AXS Inc., Madison, Wisconsin, USA.

Spek, A. L. (2003). J. Appl. Cryst. 36, 7-13. 


\section{supporting information}

Acta Cryst. (2006). E62, o2803-o2804 [https://doi.org/10.1107/S160053680602109X]

\section{2-(2-Methylphenyl)-4,5-diphenyl-1H-imidazole}

\section{T. Seethalakshmi, A. Puratchikody, Daniel E. Lynch, P. Kaliannan and S. Thamotharan}

2-(2-Methylphenyl)-4,5-diphenyl-1H-imidazole

Crystal data

$\mathrm{C}_{22} \mathrm{H}_{18} \mathrm{~N}_{2}$

$M_{r}=310.38$

Monoclinic, $C c$

Hall symbol: C $-2 \mathrm{yc}$

$a=10.7538(5) \AA$

$b=19.3999(9) \AA$

$c=8.7900(3) \AA$

$\beta=112.886(2)^{\circ}$

$V=1689.44(13) \AA^{3}$

$Z=4$

Data collection

Nonius KappaCCD area-detector diffractometer

Radiation source: Bruker Nonius FR591 rotating anode

$10 \mathrm{~cm}$ confocal mirrors monochromator

Detector resolution: 9.091 pixels $\mathrm{mm}^{-1}$

$\varphi$ and $\omega$ scans

Absorption correction: multi-scan

(SADABS; Sheldrick, 2003)

\section{Refinement}

Refinement on $F^{2}$

Least-squares matrix: full

$R\left[F^{2}>2 \sigma\left(F^{2}\right)\right]=0.041$

$w R\left(F^{2}\right)=0.103$

$S=1.06$

1945 reflections

223 parameters

2 restraints

Primary atom site location: structure-invariant direct methods

Secondary atom site location: difference Fourier map
$F(000)=656$

$D_{\mathrm{x}}=1.220 \mathrm{Mg} \mathrm{m}^{-3}$

Melting point: $484 \mathrm{~K}$

Mo $K \alpha$ radiation, $\lambda=0.71073 \AA$

Cell parameters from 1980 reflections

$\theta=2.9-27.5^{\circ}$

$\mu=0.07 \mathrm{~mm}^{-1}$

$T=120 \mathrm{~K}$

Rod, colourless

$0.36 \times 0.06 \times 0.04 \mathrm{~mm}$

$T_{\min }=0.745, T_{\max }=0.927$

10671 measured reflections

1945 independent reflections

1744 reflections with $I>2 \sigma(I)$

$R_{\text {int }}=0.059$

$\theta_{\max }=27.5^{\circ}, \theta_{\min }=3.3^{\circ}$

$h=-11 \rightarrow 13$

$k=-25 \rightarrow 25$

$l=-11 \rightarrow 11$

Hydrogen site location: inferred from neighbouring sites

$\mathrm{H}$ atoms treated by a mixture of independent and constrained refinement

$w=1 /\left[\sigma^{2}\left(F_{\mathrm{o}}^{2}\right)+(0.0523 P)^{2}+0.7664 P\right]$ where $P=\left(F_{\mathrm{o}}^{2}+2 F_{\mathrm{c}}^{2}\right) / 3$

$(\Delta / \sigma)_{\max }<0.001$

$\Delta \rho_{\max }=0.19$ e $\AA^{-3}$

$\Delta \rho_{\min }=-0.17$ e $\AA^{-3}$

Extinction correction: SHELXL97, $\mathrm{Fc}^{*}=\mathrm{kFc}\left[1+0.001 \times \mathrm{Fc}^{2} \lambda^{3} / \sin (2 \theta)\right]^{-1 / 4}$

Extinction coefficient: 0.0089 (16) 


\section{Special details}

Geometry. All e.s.d.'s (except the e.s.d. in the dihedral angle between two 1.s. planes) are estimated using the full covariance matrix. The cell e.s.d.'s are taken into account individually in the estimation of e.s.d.'s in distances, angles and torsion angles; correlations between e.s.d.'s in cell parameters are only used when they are defined by crystal symmetry. An approximate (isotropic) treatment of cell e.s.d.'s is used for estimating e.s.d.'s involving 1.s. planes.

Refinement. Refinement of $F^{2}$ against ALL reflections. The weighted $R$-factor $w R$ and goodness of fit $S$ are based on $F^{2}$, conventional $R$-factors $R$ are based on $F$, with $F$ set to zero for negative $F^{2}$. The threshold expression of $F^{2}>\sigma\left(F^{2}\right)$ is used only for calculating $R$-factors (gt) etc. and is not relevant to the choice of reflections for refinement. $R$-factors based on $F^{2}$ are statistically about twice as large as those based on $F$, and $R$ - factors based on ALL data will be even larger.

Fractional atomic coordinates and isotropic or equivalent isotropic displacement parameters $\left(\AA^{2}\right)$

\begin{tabular}{|c|c|c|c|c|}
\hline & $x$ & $y$ & $z$ & $U_{\text {iso }} * / U_{\text {eq }}$ \\
\hline N1 & $0.7086(2)$ & $0.01344(11)$ & $0.0732(2)$ & $0.0210(4)$ \\
\hline $\mathrm{H} 1$ & $0.698(3)$ & $-0.0160(15)$ & $-0.007(4)$ & $0.019(7)^{*}$ \\
\hline N3 & $0.6705(2)$ & $0.06587(10)$ & $0.2743(2)$ & $0.0211(4)$ \\
\hline $\mathrm{C} 2$ & $0.6360(2)$ & $0.01323(12)$ & $0.1697(3)$ & $0.0204(5)$ \\
\hline $\mathrm{C} 4$ & $0.7688(2)$ & $0.10230(12)$ & 0.2418 & $0.0204(5)$ \\
\hline $\mathrm{C} 5$ & $0.7926(3)$ & $0.07057(12)$ & $0.1148(3)$ & $0.0215(5)$ \\
\hline $\mathrm{C} 21$ & $0.5397(3)$ & $-0.04191(13)$ & 0.1607 (3) & $0.0241(5)$ \\
\hline $\mathrm{C} 22$ & $0.4158(3)$ & $-0.02859(15)$ & 0.1729 & $0.0292(6)$ \\
\hline $\mathrm{C} 23$ & $0.3333(3)$ & $-0.08493(16)$ & $0.1644(4)$ & $0.0398(7)$ \\
\hline $\mathrm{H} 23$ & 0.2486 & -0.0772 & 0.1719 & $0.048 *$ \\
\hline $\mathrm{C} 24$ & $0.3697(4)$ & $-0.15116(17)$ & 0.1458 & $0.0455(8)$ \\
\hline $\mathrm{H} 24$ & 0.3110 & -0.1884 & 0.1415 & $0.055^{*}$ \\
\hline $\mathrm{C} 25$ & $0.4917(4)$ & $-0.16377(14)$ & $0.1332(4)$ & $0.0393(7)$ \\
\hline $\mathrm{H} 25$ & 0.5173 & -0.2096 & 0.1201 & $0.047 *$ \\
\hline $\mathrm{C} 26$ & $0.5763(3)$ & $-0.10916(14)$ & 0.1399 & $0.0292(6)$ \\
\hline $\mathrm{H} 26$ & 0.6600 & -0.1175 & 0.1302 & $0.035^{*}$ \\
\hline $\mathrm{C} 27$ & 0.3689 & $0.04324(16)$ & $0.1896(4)$ & $0.0361(7)$ \\
\hline $\mathrm{H} 271$ & 0.2753 & 0.0415 & 0.1807 & $0.054^{*}$ \\
\hline $\mathrm{H} 272$ & 0.3745 & 0.0725 & 0.1016 & $0.054 *$ \\
\hline $\mathrm{H} 273$ & 0.4265 & 0.0623 & 0.2973 & $0.054 *$ \\
\hline C41 & 0.8327 & $0.16304(12)$ & 0.3411 & $0.0220(5)$ \\
\hline $\mathrm{C} 42$ & $0.9678(3)$ & $0.18026(13)$ & $0.3771(3)$ & $0.0261(5)$ \\
\hline H42 & 1.0207 & 0.1520 & 0.3372 & $0.031 *$ \\
\hline $\mathrm{C} 43$ & $1.0247(3)$ & $0.23823(14)$ & $0.4704(3)$ & $0.0320(6)$ \\
\hline H43 & 1.1161 & 0.2496 & 0.4930 & $0.038 *$ \\
\hline $\mathrm{C} 44$ & $0.9492(3)$ & $0.27985(13)$ & $0.5311(3)$ & $0.0329(6)$ \\
\hline H44 & 0.9885 & 0.3197 & 0.5944 & $0.039 *$ \\
\hline $\mathrm{C} 45$ & $0.8167(3)$ & $0.26271(14)$ & $0.4986(3)$ & $0.0312(6)$ \\
\hline H45 & 0.7646 & 0.2909 & 0.5399 & $0.037 *$ \\
\hline $\mathrm{C} 46$ & $0.7595(3)$ & $0.20471(13)$ & $0.4064(3)$ & $0.0260(6)$ \\
\hline H46 & 0.6688 & 0.1930 & 0.3870 & $0.031 *$ \\
\hline $\mathrm{C} 51$ & $0.8762(2)$ & $0.08675(13)$ & $0.0212(3)$ & $0.0230(5)$ \\
\hline $\mathrm{C} 52$ & 0.9424 & $0.03489(15)$ & -0.0275 & $0.0277(6)$ \\
\hline H52 & 0.9372 & -0.0114 & 0.0048 & $0.033^{*}$ \\
\hline $\mathrm{C} 53$ & $1.0161(3)$ & $0.05019(16)$ & $-0.1228(3)$ & $0.0325(6)$ \\
\hline
\end{tabular}


supporting information

\begin{tabular}{lllll} 
H53 & 1.0609 & 0.0145 & -0.1556 & $0.039^{*}$ \\
C54 & $1.0237(3)$ & $0.11818(17)$ & $-0.1700(4)$ & $0.0379(7)$ \\
H54 & 1.0732 & 0.1289 & -0.2360 & $0.045^{*}$ \\
C55 & $0.9597(3)$ & $0.16969(15)$ & $-0.1210(3)$ & $0.0345(7)$ \\
H55 & 0.9658 & 0.2160 & -0.1528 & $0.041^{*}$ \\
C56 & $0.8860(3)$ & $0.15480(14)$ & $-0.0255(3)$ & $0.0288(6)$ \\
H56 & 0.8425 & 0.1908 & 0.0081 & $0.035^{*}$ \\
\hline
\end{tabular}

Atomic displacement parameters $\left(\AA^{2}\right)$

\begin{tabular}{lllllll}
\hline & $U^{11}$ & $U^{22}$ & $U^{33}$ & $U^{12}$ & $U^{13}$ & $U^{23}$ \\
\hline $\mathrm{N} 1$ & $0.0242(11)$ & $0.0209(10)$ & $0.0183(10)$ & $-0.0017(8)$ & $0.0088(9)$ & $-0.0005(8)$ \\
$\mathrm{N} 3$ & $0.0226(10)$ & $0.0208(9)$ & $0.0197(9)$ & $-0.0028(8)$ & $0.0082(8)$ & $0.0007(8)$ \\
$\mathrm{C} 2$ & $0.0231(13)$ & $0.0205(11)$ & $0.0174(11)$ & $0.0003(10)$ & $0.0079(10)$ & $0.0029(9)$ \\
$\mathrm{C} 4$ & $0.0187(12)$ & $0.0208(11)$ & $0.0211(11)$ & $-0.0001(9)$ & $0.0072(9)$ & $0.0024(9)$ \\
$\mathrm{C} 5$ & $0.0242(13)$ & $0.0202(11)$ & $0.0209(11)$ & $-0.0007(10)$ & $0.0096(10)$ & $0.0035(9)$ \\
$\mathrm{C} 21$ & $0.0281(14)$ & $0.0246(12)$ & $0.0186(11)$ & $-0.0051(10)$ & $0.0081(10)$ & $0.0013(10)$ \\
$\mathrm{C} 22$ & $0.0258(14)$ & $0.0348(15)$ & $0.0256(12)$ & $-0.0046(11)$ & $0.0086(11)$ & $0.0047(11)$ \\
$\mathrm{C} 23$ & $0.0326(17)$ & $0.0443(17)$ & $0.0429(17)$ & $-0.0126(14)$ & $0.0152(14)$ & $0.0029(14)$ \\
$\mathrm{C} 24$ & $0.045(2)$ & $0.0362(16)$ & $0.0511(19)$ & $-0.0205(14)$ & $0.0139(16)$ & $0.0030(14)$ \\
$\mathrm{C} 25$ & $0.051(2)$ & $0.0252(14)$ & $0.0413(16)$ & $-0.0104(13)$ & $0.0182(15)$ & $-0.0013(12)$ \\
$\mathrm{C} 26$ & $0.0356(16)$ & $0.0253(13)$ & $0.0271(13)$ & $-0.0044(11)$ & $0.0128(12)$ & $-0.0005(10)$ \\
$\mathrm{C} 27$ & $0.0258(14)$ & $0.0404(16)$ & $0.0416(16)$ & $0.0003(13)$ & $0.0127(13)$ & $0.0006(13)$ \\
$\mathrm{C} 41$ & $0.0257(13)$ & $0.0193(11)$ & $0.0203(10)$ & $-0.0003(10)$ & $0.0083(10)$ & $0.0040(9)$ \\
$\mathrm{C} 42$ & $0.0225(13)$ & $0.0246(12)$ & $0.0282(13)$ & $-0.0004(10)$ & $0.0064(11)$ & $0.0020(10)$ \\
$\mathrm{C} 43$ & $0.0265(15)$ & $0.0277(14)$ & $0.0343(14)$ & $-0.0045(11)$ & $0.0037(12)$ & $0.0024(11)$ \\
$\mathrm{C} 44$ & $0.0367(17)$ & $0.0187(12)$ & $0.0320(14)$ & $-0.0042(11)$ & $0.0011(12)$ & $-0.0036(11)$ \\
$\mathrm{C} 45$ & $0.0353(16)$ & $0.0260(13)$ & $0.0292(13)$ & $0.0028(12)$ & $0.0090(12)$ & $-0.0010(11)$ \\
$\mathrm{C} 46$ & $0.0254(14)$ & $0.0252(13)$ & $0.0243(11)$ & $0.0002(11)$ & $0.0061(10)$ & $-0.0017(10)$ \\
$\mathrm{C} 51$ & $0.0210(13)$ & $0.0287(13)$ & $0.0200(11)$ & $-0.0061(11)$ & $0.0089(10)$ & $-0.0013(10)$ \\
$\mathrm{C} 52$ & $0.0237(13)$ & $0.0346(14)$ & $0.0238(12)$ & $-0.0061(11)$ & $0.0081(10)$ & $-0.0051(11)$ \\
$\mathrm{C} 53$ & $0.0267(15)$ & $0.0464(17)$ & $0.0285(13)$ & $-0.0046(13)$ & $0.0150(12)$ & $-0.0101(12)$ \\
C54 & $0.0345(17)$ & $0.0562(19)$ & $0.0308(14)$ & $-0.0161(14)$ & $0.0212(13)$ & $-0.0070(14)$ \\
C55 & $0.0372(16)$ & $0.0388(16)$ & $0.0282(13)$ & $-0.0114(13)$ & $0.0136(12)$ & $0.0046(12)$ \\
C56 & $0.0297(15)$ & $0.0290(13)$ & $0.0286(13)$ & $-0.0034(11)$ & $0.0123(12)$ & $0.0038(11)$ \\
& & & & & & \\
\hline & & & & & &
\end{tabular}

Geometric parameters $\left(A,{ }^{\circ}\right)$

\begin{tabular}{llll}
\hline $\mathrm{N} 1-\mathrm{C} 2$ & $1.358(3)$ & $\mathrm{C} 41-\mathrm{C} 46$ & $1.398(4)$ \\
$\mathrm{N} 1-\mathrm{C} 5$ & $1.386(3)$ & $\mathrm{C} 41-\mathrm{C} 42$ & $1.401(4)$ \\
$\mathrm{N} 1-\mathrm{H} 1$ & $0.88(3)$ & $\mathrm{C} 42-\mathrm{C} 43$ & $1.386(4)$ \\
$\mathrm{N} 3-\mathrm{C} 2$ & $1.327(3)$ & $\mathrm{C} 42-\mathrm{H} 42$ & 0.9500 \\
$\mathrm{~N} 3-\mathrm{C} 4$ & $1.390(3)$ & $\mathrm{C} 43-\mathrm{C} 44$ & $1.390(4)$ \\
$\mathrm{C} 2-\mathrm{C} 21$ & $1.470(3)$ & $\mathrm{C} 43-\mathrm{H} 43$ & 0.9500 \\
$\mathrm{C} 4-\mathrm{C} 5$ & $1.383(3)$ & $\mathrm{C} 44-\mathrm{C} 45$ & $1.381(4)$ \\
$\mathrm{C} 4-\mathrm{C} 41$ & $1.468(3)$ & $\mathrm{C} 44-\mathrm{H} 44$ & 0.9500 \\
$\mathrm{C} 5-\mathrm{C} 51$ & $1.469(3)$ & $\mathrm{C} 45-\mathrm{C} 46$ & $1.384(4)$ \\
$\mathrm{C} 21-\mathrm{C} 26$ & $1.395(4)$ & $\mathrm{C} 45-\mathrm{H} 45$ & 0.9500
\end{tabular}




\begin{tabular}{|c|c|c|c|}
\hline $\mathrm{C} 21-\mathrm{C} 22$ & $1.401(4)$ & $\mathrm{C} 46-\mathrm{H} 46$ & 0.9500 \\
\hline $\mathrm{C} 22-\mathrm{C} 23$ & $1.391(4)$ & $\mathrm{C} 51-\mathrm{C} 52$ & $1.393(4)$ \\
\hline $\mathrm{C} 22-\mathrm{C} 27$ & $1.508(4)$ & $\mathrm{C} 51-\mathrm{C} 56$ & $1.399(3)$ \\
\hline $\mathrm{C} 23-\mathrm{C} 24$ & $1.372(5)$ & $\mathrm{C} 52-\mathrm{C} 53$ & $1.391(4)$ \\
\hline $\mathrm{C} 23-\mathrm{H} 23$ & 0.9500 & $\mathrm{C} 52-\mathrm{H} 52$ & 0.9500 \\
\hline $\mathrm{C} 24-\mathrm{C} 25$ & $1.380(5)$ & $\mathrm{C} 53-\mathrm{C} 54$ & $1.395(4)$ \\
\hline $\mathrm{C} 24-\mathrm{H} 24$ & 0.9500 & C53-H53 & 0.9500 \\
\hline $\mathrm{C} 25-\mathrm{C} 26$ & $1.383(4)$ & $\mathrm{C} 54-\mathrm{C} 55$ & $1.374(4)$ \\
\hline $\mathrm{C} 25-\mathrm{H} 25$ & 0.9500 & C54-H54 & 0.9500 \\
\hline $\mathrm{C} 26-\mathrm{H} 26$ & 0.9500 & $\mathrm{C} 55-\mathrm{C} 56$ & $1.391(4)$ \\
\hline $\mathrm{C} 27-\mathrm{H} 271$ & 0.9800 & C55-H55 & 0.9500 \\
\hline C27-H272 & 0.9800 & C56-H56 & 0.9500 \\
\hline $\mathrm{C} 27-\mathrm{H} 273$ & 0.9800 & & \\
\hline $\mathrm{C} 2-\mathrm{N} 1-\mathrm{C} 5$ & $108.2(2)$ & $\mathrm{C} 46-\mathrm{C} 41-\mathrm{C} 42$ & $118.0(2)$ \\
\hline $\mathrm{C} 2-\mathrm{N} 1-\mathrm{H} 1$ & $124.7(18)$ & $\mathrm{C} 46-\mathrm{C} 41-\mathrm{C} 4$ & $120.0(2)$ \\
\hline $\mathrm{C} 5-\mathrm{N} 1-\mathrm{H} 1$ & $126.9(18)$ & $\mathrm{C} 42-\mathrm{C} 41-\mathrm{C} 4$ & $122.0(2)$ \\
\hline $\mathrm{C} 2-\mathrm{N} 3-\mathrm{C} 4$ & $106.22(19)$ & $\mathrm{C} 43-\mathrm{C} 42-\mathrm{C} 41$ & $120.5(2)$ \\
\hline $\mathrm{N} 3-\mathrm{C} 2-\mathrm{N} 1$ & $110.9(2)$ & $\mathrm{C} 43-\mathrm{C} 42-\mathrm{H} 42$ & 119.7 \\
\hline $\mathrm{N} 3-\mathrm{C} 2-\mathrm{C} 21$ & $126.8(2)$ & $\mathrm{C} 41-\mathrm{C} 42-\mathrm{H} 42$ & 119.7 \\
\hline $\mathrm{N} 1-\mathrm{C} 2-\mathrm{C} 21$ & $122.2(2)$ & $\mathrm{C} 42-\mathrm{C} 43-\mathrm{C} 44$ & $120.6(3)$ \\
\hline $\mathrm{C} 5-\mathrm{C} 4-\mathrm{N} 3$ & $109.5(2)$ & $\mathrm{C} 42-\mathrm{C} 43-\mathrm{H} 43$ & 119.7 \\
\hline $\mathrm{C} 5-\mathrm{C} 4-\mathrm{C} 41$ & $130.3(2)$ & $\mathrm{C} 44-\mathrm{C} 43-\mathrm{H} 43$ & 119.7 \\
\hline $\mathrm{N} 3-\mathrm{C} 4-\mathrm{C} 41$ & $120.2(2)$ & $\mathrm{C} 45-\mathrm{C} 44-\mathrm{C} 43$ & $119.4(2)$ \\
\hline $\mathrm{C} 4-\mathrm{C} 5-\mathrm{N} 1$ & $105.3(2)$ & $\mathrm{C} 45-\mathrm{C} 44-\mathrm{H} 44$ & 120.3 \\
\hline $\mathrm{C} 4-\mathrm{C} 5-\mathrm{C} 51$ & $134.6(2)$ & $\mathrm{C} 43-\mathrm{C} 44-\mathrm{H} 44$ & 120.3 \\
\hline $\mathrm{N} 1-\mathrm{C} 5-\mathrm{C} 51$ & $120.0(2)$ & $\mathrm{C} 44-\mathrm{C} 45-\mathrm{C} 46$ & $120.3(3)$ \\
\hline $\mathrm{C} 26-\mathrm{C} 21-\mathrm{C} 22$ & $120.5(2)$ & $\mathrm{C} 44-\mathrm{C} 45-\mathrm{H} 45$ & 119.8 \\
\hline $\mathrm{C} 26-\mathrm{C} 21-\mathrm{C} 2$ & $117.3(2)$ & $\mathrm{C} 46-\mathrm{C} 45-\mathrm{H} 45$ & 119.8 \\
\hline $\mathrm{C} 22-\mathrm{C} 21-\mathrm{C} 2$ & $122.2(2)$ & $\mathrm{C} 45-\mathrm{C} 46-\mathrm{C} 41$ & $121.2(3)$ \\
\hline $\mathrm{C} 23-\mathrm{C} 22-\mathrm{C} 21$ & $117.2(3)$ & $\mathrm{C} 45-\mathrm{C} 46-\mathrm{H} 46$ & 119.4 \\
\hline $\mathrm{C} 23-\mathrm{C} 22-\mathrm{C} 27$ & $120.0(3)$ & $\mathrm{C} 41-\mathrm{C} 46-\mathrm{H} 46$ & 119.4 \\
\hline $\mathrm{C} 21-\mathrm{C} 22-\mathrm{C} 27$ & $122.8(2)$ & $\mathrm{C} 52-\mathrm{C} 51-\mathrm{C} 56$ & $119.1(2)$ \\
\hline $\mathrm{C} 24-\mathrm{C} 23-\mathrm{C} 22$ & $122.5(3)$ & $\mathrm{C} 52-\mathrm{C} 51-\mathrm{C} 5$ & $121.0(2)$ \\
\hline $\mathrm{C} 24-\mathrm{C} 23-\mathrm{H} 23$ & 118.8 & $\mathrm{C} 56-\mathrm{C} 51-\mathrm{C} 5$ & 119.9 (2) \\
\hline $\mathrm{C} 22-\mathrm{C} 23-\mathrm{H} 23$ & 118.8 & $\mathrm{C} 53-\mathrm{C} 52-\mathrm{C} 51$ & $120.7(3)$ \\
\hline $\mathrm{C} 23-\mathrm{C} 24-\mathrm{C} 25$ & $120.0(3)$ & $\mathrm{C} 53-\mathrm{C} 52-\mathrm{H} 52$ & 119.7 \\
\hline $\mathrm{C} 23-\mathrm{C} 24-\mathrm{H} 24$ & 120.0 & $\mathrm{C} 51-\mathrm{C} 52-\mathrm{H} 52$ & 119.7 \\
\hline $\mathrm{C} 25-\mathrm{C} 24-\mathrm{H} 24$ & 120.0 & $\mathrm{C} 52-\mathrm{C} 53-\mathrm{C} 54$ & $119.6(3)$ \\
\hline $\mathrm{C} 24-\mathrm{C} 25-\mathrm{C} 26$ & $119.4(3)$ & $\mathrm{C} 52-\mathrm{C} 53-\mathrm{H} 53$ & 120.2 \\
\hline $\mathrm{C} 24-\mathrm{C} 25-\mathrm{H} 25$ & 120.3 & $\mathrm{C} 54-\mathrm{C} 53-\mathrm{H} 53$ & 120.2 \\
\hline $\mathrm{C} 26-\mathrm{C} 25-\mathrm{H} 25$ & 120.3 & $\mathrm{C} 55-\mathrm{C} 54-\mathrm{C} 53$ & $120.0(2)$ \\
\hline $\mathrm{C} 25-\mathrm{C} 26-\mathrm{C} 21$ & $120.5(3)$ & $\mathrm{C} 55-\mathrm{C} 54-\mathrm{H} 54$ & 120.0 \\
\hline $\mathrm{C} 25-\mathrm{C} 26-\mathrm{H} 26$ & 119.8 & $\mathrm{C} 53-\mathrm{C} 54-\mathrm{H} 54$ & 120.0 \\
\hline $\mathrm{C} 21-\mathrm{C} 26-\mathrm{H} 26$ & 119.8 & $\mathrm{C} 54-\mathrm{C} 55-\mathrm{C} 56$ & $120.7(3)$ \\
\hline $\mathrm{C} 22-\mathrm{C} 27-\mathrm{H} 271$ & 109.5 & $\mathrm{C} 54-\mathrm{C} 55-\mathrm{H} 55$ & 119.7 \\
\hline $\mathrm{C} 22-\mathrm{C} 27-\mathrm{H} 272$ & 109.5 & $\mathrm{C} 56-\mathrm{C} 55-\mathrm{H} 55$ & 119.7 \\
\hline $\mathrm{H} 271-\mathrm{C} 27-\mathrm{H} 272$ & 109.5 & $\mathrm{C} 55-\mathrm{C} 56-\mathrm{C} 51$ & $119.9(3)$ \\
\hline
\end{tabular}




\begin{tabular}{|c|c|c|c|}
\hline $\mathrm{C} 22-\mathrm{C} 27-\mathrm{H} 273$ & 109.5 & $\mathrm{C} 55-\mathrm{C} 56-\mathrm{H} 56$ & 120.0 \\
\hline $\mathrm{H} 271-\mathrm{C} 27-\mathrm{H} 273$ & 109.5 & $\mathrm{C} 51-\mathrm{C} 56-\mathrm{H} 56$ & 120.0 \\
\hline $\mathrm{H} 272-\mathrm{C} 27-\mathrm{H} 273$ & 109.5 & & \\
\hline $\mathrm{C} 4-\mathrm{N} 3-\mathrm{C} 2-\mathrm{N} 1$ & $0.9(3)$ & $\mathrm{C} 2-\mathrm{C} 21-\mathrm{C} 26-\mathrm{C} 25$ & $178.9(2)$ \\
\hline $\mathrm{C} 4-\mathrm{N} 3-\mathrm{C} 2-\mathrm{C} 21$ & $177.1(2)$ & $\mathrm{C} 5-\mathrm{C} 4-\mathrm{C} 41-\mathrm{C} 46$ & $150.7(3)$ \\
\hline $\mathrm{C} 5-\mathrm{N} 1-\mathrm{C} 2-\mathrm{N} 3$ & $-1.7(3)$ & $\mathrm{N} 3-\mathrm{C} 4-\mathrm{C} 41-\mathrm{C} 46$ & $-31.7(3)$ \\
\hline $\mathrm{C} 5-\mathrm{N} 1-\mathrm{C} 2-\mathrm{C} 21$ & $-178.1(2)$ & $\mathrm{C} 5-\mathrm{C} 4-\mathrm{C} 41-\mathrm{C} 42$ & $-31.0(4)$ \\
\hline $\mathrm{C} 2-\mathrm{N} 3-\mathrm{C} 4-\mathrm{C} 5$ & $0.2(3)$ & $\mathrm{N} 3-\mathrm{C} 4-\mathrm{C} 41-\mathrm{C} 42$ & $146.6(2)$ \\
\hline $\mathrm{C} 2-\mathrm{N} 3-\mathrm{C} 4-\mathrm{C} 41$ & $-177.9(2)$ & $\mathrm{C} 46-\mathrm{C} 41-\mathrm{C} 42-\mathrm{C} 43$ & $-2.0(4)$ \\
\hline $\mathrm{N} 3-\mathrm{C} 4-\mathrm{C} 5-\mathrm{N} 1$ & $-1.1(3)$ & $\mathrm{C} 4-\mathrm{C} 41-\mathrm{C} 42-\mathrm{C} 43$ & $179.6(2)$ \\
\hline $\mathrm{C} 41-\mathrm{C} 4-\mathrm{C} 5-\mathrm{N} 1$ & $176.7(2)$ & $\mathrm{C} 41-\mathrm{C} 42-\mathrm{C} 43-\mathrm{C} 44$ & $0.6(4)$ \\
\hline $\mathrm{N} 3-\mathrm{C} 4-\mathrm{C} 5-\mathrm{C} 51$ & $175.4(3)$ & $\mathrm{C} 42-\mathrm{C} 43-\mathrm{C} 44-\mathrm{C} 45$ & $0.4(4)$ \\
\hline $\mathrm{C} 41-\mathrm{C} 4-\mathrm{C} 5-\mathrm{C} 51$ & $-6.8(5)$ & $\mathrm{C} 43-\mathrm{C} 44-\mathrm{C} 45-\mathrm{C} 46$ & $-0.1(4)$ \\
\hline $\mathrm{C} 2-\mathrm{N} 1-\mathrm{C} 5-\mathrm{C} 4$ & $1.7(3)$ & $\mathrm{C} 44-\mathrm{C} 45-\mathrm{C} 46-\mathrm{C} 41$ & $-1.4(4)$ \\
\hline $\mathrm{C} 2-\mathrm{N} 1-\mathrm{C} 5-\mathrm{C} 51$ & $-175.5(2)$ & $\mathrm{C} 42-\mathrm{C} 41-\mathrm{C} 46-\mathrm{C} 45$ & $2.4(4)$ \\
\hline $\mathrm{N} 3-\mathrm{C} 2-\mathrm{C} 21-\mathrm{C} 26$ & $-136.8(3)$ & $\mathrm{C} 4-\mathrm{C} 41-\mathrm{C} 46-\mathrm{C} 45$ & $-179.2(2)$ \\
\hline $\mathrm{N} 1-\mathrm{C} 2-\mathrm{C} 21-\mathrm{C} 26$ & $39.0(3)$ & $\mathrm{C} 4-\mathrm{C} 5-\mathrm{C} 51-\mathrm{C} 52$ & $142.6(3)$ \\
\hline $\mathrm{N} 3-\mathrm{C} 2-\mathrm{C} 21-\mathrm{C} 22$ & $43.0(4)$ & $\mathrm{N} 1-\mathrm{C} 5-\mathrm{C} 51-\mathrm{C} 52$ & $-41.2(3)$ \\
\hline $\mathrm{N} 1-\mathrm{C} 2-\mathrm{C} 21-\mathrm{C} 22$ & $-141.2(2)$ & $\mathrm{C} 4-\mathrm{C} 5-\mathrm{C} 51-\mathrm{C} 56$ & $-39.7(4)$ \\
\hline $\mathrm{C} 26-\mathrm{C} 21-\mathrm{C} 22-\mathrm{C} 23$ & $0.4(4)$ & $\mathrm{N} 1-\mathrm{C} 5-\mathrm{C} 51-\mathrm{C} 56$ & $136.4(3)$ \\
\hline $\mathrm{C} 2-\mathrm{C} 21-\mathrm{C} 22-\mathrm{C} 23$ & $-179.3(2)$ & $\mathrm{C} 56-\mathrm{C} 51-\mathrm{C} 52-\mathrm{C} 53$ & $-0.8(4)$ \\
\hline $\mathrm{C} 26-\mathrm{C} 21-\mathrm{C} 22-\mathrm{C} 27$ & $-177.8(2)$ & $\mathrm{C} 5-\mathrm{C} 51-\mathrm{C} 52-\mathrm{C} 53$ & $176.9(3)$ \\
\hline $\mathrm{C} 2-\mathrm{C} 21-\mathrm{C} 22-\mathrm{C} 27$ & $2.4(4)$ & $\mathrm{C} 51-\mathrm{C} 52-\mathrm{C} 53-\mathrm{C} 54$ & $0.1(4)$ \\
\hline $\mathrm{C} 21-\mathrm{C} 22-\mathrm{C} 23-\mathrm{C} 24$ & $0.3(4)$ & C52-C53-C54-C55 & $0.6(4)$ \\
\hline $\mathrm{C} 27-\mathrm{C} 22-\mathrm{C} 23-\mathrm{C} 24$ & $178.6(3)$ & $\mathrm{C} 53-\mathrm{C} 54-\mathrm{C} 55-\mathrm{C} 56$ & $-0.5(5)$ \\
\hline $\mathrm{C} 22-\mathrm{C} 23-\mathrm{C} 24-\mathrm{C} 25$ & $-0.6(5)$ & $\mathrm{C} 54-\mathrm{C} 55-\mathrm{C} 56-\mathrm{C} 51$ & $-0.3(4)$ \\
\hline $\mathrm{C} 23-\mathrm{C} 24-\mathrm{C} 25-\mathrm{C} 26$ & $0.1(5)$ & $\mathrm{C} 52-\mathrm{C} 51-\mathrm{C} 56-\mathrm{C} 55$ & $0.9(4)$ \\
\hline $\mathrm{C} 24-\mathrm{C} 25-\mathrm{C} 26-\mathrm{C} 21$ & $0.6(4)$ & $\mathrm{C} 5-\mathrm{C} 51-\mathrm{C} 56-\mathrm{C} 55$ & $-176.8(3)$ \\
\hline $\mathrm{C} 22-\mathrm{C} 21-\mathrm{C} 26-\mathrm{C} 25$ & $-0.9(4)$ & & \\
\hline
\end{tabular}

Hydrogen-bond geometry $\left(A,{ }^{\circ}\right)$

\begin{tabular}{lllll}
\hline$D-\mathrm{H} \cdots A$ & $D-\mathrm{H}$ & $\mathrm{H} \cdots A$ & $D \cdots A$ & $D-\mathrm{H} \cdots A$ \\
\hline $\mathrm{N} 1-\mathrm{H} 1 \cdots \mathrm{N} 3^{\mathrm{i}}$ & $0.88(3)$ & $2.07(3)$ & $2.933(3)$ & $168(3)$ \\
\hline
\end{tabular}

Symmetry code: (i) $x,-y, z-1 / 2$. 